\title{
The Population of Open Clusters in the Nearest kpc from the Sun
}

\author{
S. Röser ${ }^{1}$, N. V. Kharchenko ${ }^{1,2,4}$, A. E. Piskunov ${ }^{1,3,4}$, \\ E. Schilbach ${ }^{1}$ and R.-D. Scholz ${ }^{4}$ \\ ${ }^{1}$ Astronomisches Rechen-Institut, Moenchhofstr. 12-14, 69120 Heidelberg, Germany \\ email: roeser@ari.uni-heidelberg.de \\ ${ }^{2}$ Main Astronomical Observatory, Kiev, Ukraine \\ ${ }^{3}$ Institute of Astronomy of the Russian Acad. Sci., Moscow, Russia \\ ${ }^{4}$ Astrophysikalisches Institut Potsdam, Potsdam, Germany
}

\begin{abstract}
We present a volume-limited sample $(d<850$ pc) of open clusters in the Galaxy identified from our studies on galactic open clusters based on data from the all-sky catalogue ASCC-2.5 with absolute proper motions and $B, V$ magnitudes of 2.5 million stars. The astrophysical properties of this sample are discussed.
\end{abstract}

Keywords. open clusters and associations: general, Galaxy: kinematics and dynamics, techniques: photometric, astrometry

\section{The basic material}

The basic idea underlying our studies is to use a complete homogeneous sky-survey comprising astrometric, photometric, and, partially, spectroscopic data to investigate all open clusters with the same objective methods. This sky-survey is the All-Sky Compiled Catalogue of 2.5 million stars (Kharchenko 2001) with absolute proper motions in the Hipparcos system, with $B, V$ magnitudes in the Johnson photometric system, and supplemented with spectral types and radial velocities if available. The ASCC-2.5 was used to identify known open clusters and compact associations from the Lund Catalogue (Lyngå 1987), the Dias et al. (2004) on-line data collection, and the Ruprecht, Balazs \& White (1981) list of associations. In the ASCC-2.5 we found 520 of about 1700 known clusters (Kharchenko et al. 2005a), and discovered 130 new open clusters (Kharchenko et al. 2005b). A pipeline was developed to determine cluster membership based on kinematic and photometric criteria as well as to obtain a uniform set of cluster structural, kinematic and evolutionary parameters (see Kharchenko et al. 2004 and Kharchenko et al. 2005a).

\section{Spatial distribution and kinematics}

This sample of 650 open clusters from ASCC-2.5 contains a volume-limited sub-sample of 256 clusters within a distance of 850 pc from the Sun (see Piskunov et al. 2006). The symmetry plane of the clusters' distribution is determined to be at $Z_{0}=-22 \pm 4 \mathrm{pc}$, and the scale height of open clusters is only $56 \pm 3$ pc. Within the completeness limit, the total surface density and volume density in the symmetry plane are $\Sigma=114 \mathrm{kpc}^{-2}$ and $D\left(Z_{0}\right)=1015 \mathrm{kpc}^{-3}$, respectively.

From the parameters of the spatial distribution, we estimate a total number of $10^{5}$ open clusters currently in the Galactic disk. The lifetime and formation rate of clusters obtained from the age distribution of field clusters within the completeness limit are 
found to be $322 \pm 31 \mathrm{Myr}$ and $0.23 \pm 0.03 \mathrm{kpc}^{-2} \mathrm{Myr}^{-1}$, respectively. This implies a total number of cluster generations in the history of the Galaxy between 30 to 40. Assuming a typical open cluster of the Pleiades type, we derive the total surface density of disk stars passed through the phase of open cluster members to be about $4 \times 10^{6} \mathrm{kpc}^{-2}$. Compared to the local density of disk stars of about $7 \times 10^{7} \mathrm{kpc}^{-2}$, the input of open clusters into the total population of the Galactic disk is found to be less than about $10 \%$.

We determined kinematic parameters describing the basic motions of the system of open clusters in the Galactic disk: the motion with respect to the Sun, and the differential rotation around the Galactic centre. The Solar motion components $U_{\odot}(+9.44 \pm$ $1.14 \mathrm{~km} / \mathrm{s}), V_{\odot}(+11.90 \pm 0.72 \mathrm{~km} / \mathrm{s}), W_{\odot}(+7.20 \pm 0.42 \mathrm{~km} / \mathrm{s})$ were derived from the complete kinematic data of 259 clusters located within 850 pc. Oort's constants $A(+14.5 \pm$ $0.8 \mathrm{~km} / \mathrm{s} / \mathrm{kpc})$ and $B(-13.0 \pm 1.1 \mathrm{~km} / \mathrm{s} / \mathrm{kpc})$ of the Galactic rotation were computed from the proper motions $\mu_{l}, \mu_{b}$ of 581 clusters with distances $d \leqslant 2500$ pc. These results are comparable to those obtained from young field stars in the Solar neighbourhood though, the clusters cover distances which typically twice as large as Hipparcos-based samples of field stars. A combination of accurate cluster ages and kinematical parameters provides a possibility to study the temporal variation of the cosmic velocity dispersion. On average, the dispersion of each velocity component increases by a factor of two over a time of 3 Gyr (maximum age of the clusters of our sample).

Fluctuations in the spatial and velocity distributions are attributed to the existence of four open cluster complexes (OCCs) of different ages containing up to a few tens of clusters. Members in an OCC show the same kinematic behaviour, and a narrow age spread. We find, that the youngest cluster complex, OCC $1(\log t<7.9,23$ clusters), with $19^{\circ}$ inclination to the Galactic plane, is apparently a signature of Gould's Belt. The most abundant OCC 2 complex $(27$ members) has moderate age $(\log t \approx 8.45)$. The clusters of the Perseus-Auriga group ( 8 members), having the same age as OCC 2, but different kinematics, are seen in breaks between Perseus-Auriga clouds. The oldest $(\log t \approx 8.85)$ group $(9$ members) was identified due to its large motion in the Galactic anticentre direction (for a detailed discussion see Piskunov et al. 2006).

\section{Acknowledgements}

This study was supported by DFG grant 436 RUS 113/757/0-2, and RFBR grant 06-02-16379.

\section{References}

Dias, W. S., Lépine, J. R. D., Alessi, B. S., \& Moitinho, A. 2004, Open clusters and Galactic structure, Version 2.0, http://www.astro.iag.usp.br/ wilton

Kharchenko, N. V. 2001, Kinematics and Physics of Celestial Bodies 17, 409 (ASCC-2.5, Cat. $\mathrm{I} / 280 \mathrm{~A})$

Kharchenko, N. V., Piskunov, A. E., Röser, S., Schilbach, E., \& Scholz, R.-D. 2004, Astronomische Nachrichten 325,740

Kharchenko, N. V., Piskunov, A. E., Röser, S., Schilbach, E., \& Scholz, R.-D. 2005a, A\&4A 438, 1163

Kharchenko, N. V., Piskunov, A. E., Röser, S., Schilbach, E., \& Scholz, R.-D. 2005b, A\&̛A 440, 403

Lyngå, G. 1987, Catalogue of open clusters data, Fifth edition, CDS, Strasbourg (Cat. VII/92)

Piskunov, A. E., Kharchenko, N. V., Röser, S., Schilbach, E., \& Scholz, R.-D. 2006, A $\& A 445$, 545

Ruprecht, J., Balazs, B., \& White, R. E. 1981, Catalogue of Star Clusters and Associations, Supplement 1, Associations, Akademiai Kiado, Publ. House Hungarian Acad. Sciences, Budapest 